\title{
Parametric Resonance in the Rayleigh-Duffing Oscillator with Time-Delayed Feedback
}

\author{
M. Siewe Siewe ${ }^{1,2}$, C. Tchawoua ${ }^{1}$, S. Rajasekar ${ }^{3}$ \\ ${ }^{1}$ Laboratoire de Mécanique, Département de Physique, Faculté des sciences, \\ University of Yaounde I, Box 812, Yaounde, Cameroon \\ ${ }^{2}$ Department of Electrical, Electronic and Computer Engineering, \\ University of Pretoria, Pretoria, 0002 South Africa. \\ 3 School of Physics, Bharathidasan University, Tiruchirapalli 620 024, \\ Tamilnadu, India.
}

email: martinsiewesiewe@yhoo.fr, ctchawa@yahoo.fr, rajasekar@cnld.bdu.ac.in

\begin{abstract}
We investigate the principal parametric resonance of a Rayleigh-Duffing oscillator with time-delayed feedback position and linear velocity terms. Using the asymptotic perturbation method, we obtain two slow flow equations on the amplitude and phase of the oscillator. We study the effects of the frequency detuning, the deterministic amplitude, and the time-delay on the dynamical behaviors, such as stability and bifurcation associated with the principal parametric resonance. Moreover, the appropriate choice of the feedback gain and the time-delay is discussed from the viewpoint of vibration control. It is found that the appropriate choice of the time-delay can broaden the stable region of the non-trivial steady-state solutions and enhance the control performance.
\end{abstract}

\section{Introduction}

Since the work of Verhulst (1804-1849) in population dynamics, delay differential equations (DDEs), are used to model dynamical systems in many scientific and engineering domains, e.g., population dynamics, neural networks, chemistry, climatology, economy, and so on [1]. In particular, many biological systems and controlled mechanic systems call for a description by means of DDEs. In practice, unavoidable time-delays frequently appear in the controlled mechanical or structural systems, especially in hydraulic actuators used in active suspensions of ground vehicles and active tendons of tall buildings.

Great attention has been paid to the dynamics of DDEs. It has been found that the time-delays not only make the systems retarded, but also are often sources of birth of limit cycle, lose of stability, bifurcations and chaos [2]. So there is a growing need to deepen our understanding on their complexity induced, particularly when multiple time-delays are present. As the simplest case, the systems with single time-delay have been intensively studied. Shayer and Campbell [3] and Liao and Chen [4] studied the Hopf bifurcation and double-Hopf bifurcation in DDEs. Xu and $\mathrm{Yu}$ [5] investigated the delay-induced bifurcation in a non-autonomous system with time-delayed velocity feedback. In Ref. [6], the authors 
studied the effect of time-delay on the collective dynamics of coupled limit cycle oscillators at Hopf bifurcation. Since Mackay and Glass [7] firstly found chaos in time-delayed systems, there has been increasing interest in studying the chaotic behaviour of DDEs.

Parametric excitation occurs in a wide variety of engineering applications. Here are a few recent applications: Aubin et al [8] and Zalalutdinov et al [9] found that MEMS/NEMS devices are parametrically excited when illuminated within an interference field of a continuous wave laser; Stepan et al [10] and Kalmar-Nagy [11] investigated parametric excitation in high-speed milling applications; Ramani et al [12] utilized parametric excitation to model a towed mass underwater for application to submarine dynamics; Wirkus et al [13] observed parametric excitation in the pumping of a swing; Yu [22] realized parametric excitation in a nanowire system using an oscillating electric field; Zhang et al [15] noticed parametric excitation in mass-loaded string systems such as elevators, cranes and cable-stayed bridges.

Over the last years considerable interest has been paid to the study of combined effect of parametric excitation and time-delayed control on dynamical systems. Xin-Ye et al [16] demonstrated that in the dynamical behaviour of a parametrically excited Duffing-van der Pol oscillator under linear-plus-nonlinear state feedback control with a time-delay, nontrivial steady state responses may lose their stability by a saddle-node or Hopf bifurcation as parameters vary. Attilio [17] analyzed the effect of time-delay and feedback gain from the viewpoint of vibration control and used energy considerations to study the existence and characteristics of limit cycles of the slow-flow equations of a parametrically excited van der Pol oscillator. Simohamed and Mohamed [18] investigated the interaction effect of fast vertical parametric excitation and time-delay on self-oscillation in the van der Pol oscillator. It was shown that vertical parametric excitation, in the presence of time-delay, can suppress self-excited vibrations.

The main objective of this paper is to study the dynamics of a parametrically excited Rayleigh-Duffing oscillator with time-delayed feedback position and linear velocity terms. This system is well known for its relevance to many applications, for example, vehicle dynamics, flutter of plates and shells, oil-film journal bearings, and so on. A lowest-order approximate solution of the model oscillator is constructed using the asymptotic perturbation (AP) [19]. The AP method is based on the method of averaging. The amplitude peak of the parametric resonance can be reduced by means of a correct choice of the time-delay and the feedback gain. We discuss how the existence region of steady-state solutions is modified by the feedback control and show the existence regions of the nontrivial solution in the plane of the parametric excitation amplitude and the detuning parameter for an uncontrolled system, a controlled system without time-delay, and those with time-delays corresponding to the minimum and maximum values of an appropriate equivalent damping. A bifurcation analysis and parametric excitation-response and frequency-response curves are presented. Then we perform a stability analysis of the bifurcation of the model and derive sufficient conditions for stable nontrivial solutions in order to exclude the presence of modulated motion. 


\section{Controlling parametric resonance in a system with position and velocity time-delay}

The mathematical model of the dissipative parametric Rayleigh-Duffing oscillator with two kinds of time-delayed coupling considered in this paper is as follows:

$$
\ddot{x}-\varepsilon \mu\left(1-\dot{x}^{2}\right) \dot{x}+(\delta+\varepsilon \alpha \cos t) x+\varepsilon \lambda x^{3}-\varepsilon \beta x(t-\tau)-\varepsilon \gamma \dot{x}(t-\tau)=0,
$$

where $\varepsilon$ is a positive parameter, $\mu, \delta, \alpha, \lambda, \beta$, and $\gamma$ are constants and $\tau$ is the time-delay parameter. It has been shown that $[20,21]$ (and references therein) in the absence of timedelay and parametric force, Eq. (1) can approximate the dynamics of the real quarter-car vehicle. Parametric excitation appears by periodic variations of one or more parameters in the vibration system, such as the stiffness or the damping. Among these parameters, the stiffness of the system is closely related to the natural frequency of the system and therefore has a great effect on the self-excited vibration [22]. When concerned with the reduction of amplitude vibration in mechanical systems, it is well known that all controllers exhibit a certain time-delay during operation. Most controlled mechanical systems are found to exhibit time-delay [23]. In the system (1) we consider time-delay in both linear position and velocity terms. We detune-off of $2: 1$ resonance by setting $\delta=1 / 4+\varepsilon \delta_{1}$. According to the method of averaging [19], in the absence of the parameter $\varepsilon$, Eq. (1) reduces to $\ddot{x}+x / 4=0$, with the solution:

$$
x=\rho \cos \left(\frac{1}{2} t+\varphi\right), \quad \dot{x}=-\frac{\rho}{2} \sin \left(\frac{1}{2} t+\varphi\right) .
$$

For $\varepsilon \neq 0$ (small enough) let $\rho$ and $\varphi$ be unknown functions of time $t$ in (1). Differentiating Eqs. (2) with respect to time and substituting them in (1) we get a system of equations which we solve for $\dot{\rho}(t)$ and $\rho \dot{\varphi}(t)$ to obtain

$$
\begin{aligned}
\dot{\rho} & =-\varepsilon \sin \left(\frac{1}{2} t+\varphi\right) G, \\
\rho \dot{\varphi} & =-\varepsilon \cos \left(\frac{1}{2} t+\varphi\right) G,
\end{aligned}
$$

where

$$
G=\mu\left(1-\dot{x}^{2}\right) \dot{x}-\left(\delta_{1}+\alpha \cos t\right) x-\lambda x^{3}+\beta x(t-\tau)+\gamma \dot{x}(t-\tau) .
$$

For small $\varepsilon, \dot{\rho}$ and $\dot{\varphi}$ are small. Using the method of averaging, replacing the right-hand sides of Eqs. (3) by their averages over one period of the system with $\varepsilon=0$ we obtain

$$
\begin{aligned}
\dot{\rho} & =-\frac{\varepsilon}{2 \pi} \int_{0}^{2 \pi} \sin \left(\frac{1}{2} t+\varphi\right) G d t, \\
\rho \dot{\varphi} & =-\frac{\varepsilon}{2 \pi} \int_{0}^{2 \pi} \cos \left(\frac{1}{2} t+\varphi\right) G d t
\end{aligned}
$$


in which

$$
\begin{aligned}
G=- & \frac{1}{2} \mu \rho\left[1-\frac{\rho^{2}}{4} \sin ^{2}\left(\frac{1}{2} t+\varphi\right)\right] \sin \left(\frac{1}{2} t+\varphi\right) \\
& -\left(\delta_{1}+\alpha \cos t\right) \rho \cos \left(\frac{1}{2} t+\varphi\right) \\
& -\lambda \rho^{3} \cos ^{3}\left(\frac{1}{2} t+\varphi\right)+\beta \tilde{\rho} \cos \left(\frac{1}{2}(t-\tau)+\tilde{\varphi}\right) \\
& -\frac{1}{2} \gamma \tilde{\rho} \sin \left(\frac{1}{2}(t-\tau)+\tilde{\varphi}\right),
\end{aligned}
$$

where $\tilde{\rho}=\rho(t-\tau)$ and $\tilde{\varphi}=\varphi(t-\tau)$. Evaluating the integrals $\int_{0}^{2 \pi} \sin \left(\frac{1}{2} t+\varphi\right) G d t$ and $\int_{0}^{2 \pi} \cos \left(\frac{1}{2} t+\varphi\right) G d t$, the corresponding equations are in the following form:

$$
\begin{aligned}
\dot{\rho}=\frac{\varepsilon}{4} & {\left[\mu \rho\left(1-\frac{3 \rho^{2}}{16}\right)+\alpha \rho \sin 2 \varphi-2 \beta \tilde{\rho} \sin (\varphi-\tilde{\varphi}+\tau / 2)\right.} \\
& +\gamma \tilde{\rho} \cos (\varphi-\tilde{\varphi}+\tau / 2)], \\
\rho \dot{\varphi}=\frac{\varepsilon}{4} & {\left[2 \delta_{1} \rho+\frac{3 \lambda \rho^{3}}{2}+\alpha \rho \cos 2 \varphi-2 \beta \tilde{\rho} \cos (\varphi-\tilde{\varphi}+\tau / 2)\right.} \\
& +\gamma \tilde{\rho} \sin (\varphi-\tilde{\varphi}+\tau / 2)] .
\end{aligned}
$$

Equations (7) show that $\dot{\rho}$ and $\dot{\varphi}$ are $O(\varepsilon)$. We now Taylor expand $\tilde{\rho}$ and $\tilde{\varphi}$ :

$$
\begin{aligned}
& \tilde{\rho}=\rho(t-\tau)=\rho(t)-\tau \dot{\rho}(t)+\tau^{2} \ddot{\rho}(t)+\ldots, \\
& \tilde{\varphi}=\varphi(t-\tau)=\varphi(t)-\tau \dot{\varphi}(t)+\tau^{2} \ddot{\varphi}(t)+\ldots .
\end{aligned}
$$

Equations (8) indicate that we can replace $\tilde{\rho}$ and $\tilde{\varphi}$ by $\rho$ and $\varphi$ in Eq. (7) since $\dot{\rho}, \dot{\varphi}$ and $\ddot{\rho}, \ddot{\varphi}$ are $O(\varepsilon)$ and $O\left(\varepsilon^{2}\right)$ respectively. This reduces an infinite-dimensional problem in functional analysis to a finite-dimensional problem by assuming the product $\varepsilon \tau$ is small. If we let $t=\tilde{t} / \varepsilon$ be the new time scale, we have the following averaged equations:

$$
\begin{aligned}
\dot{\rho} & =\frac{\rho}{4}\left[\mu\left(1-\frac{3 \rho^{2}}{16}\right)+\alpha \sin 2 \varphi-2 \beta \sin (\tau / 2)+\gamma \cos (\tau / 2)\right], \\
\rho \dot{\varphi} & =\frac{\rho}{4}\left[2 \delta_{1}+\frac{3 \lambda \rho^{2}}{2}+\alpha \cos 2 \varphi-2 \beta \cos (\tau / 2)+\gamma \sin (\tau / 2)\right] .
\end{aligned}
$$

The equilibrium points of system Eq. (9), obtained from a set of nonlinear algebraic equations by letting the right-hand sides of Eq. (9) be zero, corresponding to the steady state responses of the system Eq. (1). A trivial solution is $\rho=0$. When $\rho \neq 0$, we obtain

$$
\rho_{0}^{4}-2 P \rho_{0}^{2}+Q=0
$$


and

$$
\rho_{0}=\sqrt{P \pm \sqrt{P^{2}-Q}}
$$

where

$$
\begin{aligned}
P= & \frac{2}{3\left(\lambda^{2}+\frac{\mu^{2}}{64}\right)}\left[\left(2 \beta \lambda+\frac{\mu \gamma}{8}\right) \cos (\tau / 2)\right. \\
& \left.-\left(\gamma \lambda+\frac{\beta \mu}{4}\right) \sin (\tau / 2)+\frac{\mu^{2}}{8}-2 \lambda \delta_{1}\right], \\
Q= & \frac{4}{9\left(\lambda^{2}+\frac{\mu^{2}}{64}\right)}\left[K-4 \beta \gamma \sin \tau+4\left(\gamma \delta_{1}-\beta \mu\right) \sin (\tau / 2)\right. \\
& \left.\quad+2\left(\gamma \mu-4 \beta \delta_{1}\right) \cos (\tau / 2)\right], \\
K= & \mu^{2}+4 \delta_{1}^{2}+4 \beta^{2}+\gamma^{2}-\alpha^{2} .
\end{aligned}
$$

Equation (11) requires $Q>0, P>0, P^{2}>Q$ or $Q<0$. In the boundary of parameter space where the control of the amplitude is efficient, the amplitude of the controlled system $\rho_{0 c}$ (i.e. when $\beta \neq 0$ and $\gamma \neq 0$ ) is equal to that of the uncontrolled system $\rho_{0 u}$ (i.e. when $\beta=0$ and $\gamma=0$ ). Using these conditions, from Eq. (11), the boundary separating the domain where the control is efficient (reduction in the amplitude of oscillation) to the domain where it is inefficient is given by

$$
\alpha_{c}^{2}=\frac{1}{\left(P_{c}+P_{u}\right)^{2}}\left[\left(\mu^{2}+4 \delta_{1}^{2}\right)\left(P_{c}+P_{u}\right)^{2}+Q_{1}\left(P_{4}^{2}+P_{u} P_{c}\right)+\frac{Q_{1}^{2}}{9\left(\lambda^{2}+\frac{\mu^{2}}{64}\right)}\right],
$$

where $P_{u}$ and $P_{c}$ are respectively the function corresponding the uncontrolled and controlled system. $Q_{1}$ is given by

$$
\begin{aligned}
Q_{1}= & \frac{4}{9\left(\lambda^{2}+\frac{\mu^{2}}{64}\right)}\left[4 \beta^{2}+\gamma^{2}-4 \beta \gamma \sin \tau\right. \\
& \left.\quad+4\left(\gamma \delta_{1}-\beta \mu\right) \sin (\tau / 2)+2\left(\gamma \mu-4 \beta \delta_{1}\right) \cos (\tau / 2)\right] .
\end{aligned}
$$

From Fig. 1 it is clear that the critical value of the parametric amplitude excitation $\alpha$ is lower in the case where the control gain parameters verify the following inequality $\gamma>\beta$. The domain located below the curve is where the control is efficient in reducing the amplitude of the oscillations. This consideration could be applied to reduce the amplitude vibration in the quater-car model. We have plotted in Fig. 2 the frequency- and force-response curves and the results obtained in Fig. 1 are clearly verified. In fact, when the amplitude of the parametric excitation $\alpha$ is below the boundary domain obtained in Fig. 1, we see that the steady-state response of the system is lower. Also, the surface amplitude is plotted in Fig. 3 for two 
different values of control gain parameter $\beta$. Fig. 3(a) corresponds to $\beta=0.1$ and $\gamma=0.25$ while Fig. 3(b) is for $\beta=0.3$ and $\gamma=0.25$. From Fig. 3(a), it is seen that the maximum peak amplitude of the steady-state solutions are lower compared to the one in Fig. 3(b). This Figure also confirms our technical control in reducing the amplitude vibration. From the map given by Fig. 3, one can see that there are a range of time delay $\tau$ and detuning parameters $\delta_{1}$ where the steady-state solution is zero. This can be the consequence of noise effect.

In the next section, we study separately the effects of gain parameters, parametric excitation, and combined gain parameters and parametric excitation in the response of our model amplitude.

\section{Effects of gain parameters and parametric excitation in nonlin- ear resonance}

\subsection{Effects of parametric excitation alone}

We have plotted in Fig. 4, the frequency- and force-responses of the nontrivial solution when the time-delay $\tau$ is set equal to zero but the control gain parameters $\beta$ and $\gamma$ are different from zero. In Fig. 4(a), the effect of variation of amplitude of the parametric excitation is assessed. Blue color curve (lower curve) corresponds to nontrivial stable solution while the red color curve (upper curve) is the nontrivial unstable steady-state solution. For fixed parametric amplitude, two symmetric branches appear around the origin. The reduction effect due to parametric amplitude is quite important and is found to be visible only in a small region of the detuning parameter surrounding the origin. The effect of increasing of the parametric amplitude also changes and increases the instability region. The result of force-response is given in Fig. 4(b) for two values of the detuning parameters. Hopf bifurcation with multi-solution occurs as the detuning parameter $\delta_{1}$ increases. The saddle-node bifurcation disappears for the lower values of the detuning parameter.

\subsection{Effects of control gain parameters in the absence of parametric excitation}

Figures 5 represent the frequency-response curves of the system for three different situations for fixed parameters. Figure 5(a) is the frequency-response of the system when $\alpha=0$ and $\gamma=0$. Figure 5(b) is the frequency-responses for $\beta=0$ and $\alpha=0$. In Figs. 5(a) and (b), we find that the two control gain parameters increases the region of the detuning parameter surrounding the origin which is more visible when the time-delayed position is considered alone than the case where the time-delayed velocity is considered alone. As the detuning parameter increases to a large value, the peak of amplitude is greater in the case of timedelayed position and the domain of instability in red color also increase. 


\subsection{Effects of combined control gain parameters and parametric excitation}

Figure 6 shows the plot of $\rho_{0}$ as a function of $\delta_{1}$ and $\alpha$ for the system with both parametric excitation, control gain parameters $\beta$ and $\gamma$, and time-delay $\tau$. Similar comments obtained in Fig. 4 can be developed in this case. One can notice in Figs. 4, 5 and 6 studied above that an appropriate choice of the control gain parameter can reduce the response amplitude.

\section{Stability of the nontrivial steady-state solution}

The stability properties of the steady state solutions Eq. (11) obtained by plotting Figures 4,5 , and 6 are examined by applying the well-known method of linearization. We superpose small perturbations in the steady-state solution and the resulting equations are then linearized. Subsequently, we consider the eigenvalues of the corresponding system of first-order differential equations with constant coefficients (the Jacobian matrix). A positive real root indicates an unstable solution, whereas if the real parts of the eigenvalues are all negative then the steady-state solution is stable. When the real part of an eigenvalue is zero, a bifurcation occurs. A change from complex roots with negative real parts to complex roots with positive real parts would indicate the presence of a supercritical or subcritical Hopf bifurcation. The question of which possibility actually occurs depends on the nonlinear terms. The eigenvalues of the Jacobian matrix satisfy the equation

$$
\xi^{2}+p \xi+q=0,
$$

where

$$
\begin{aligned}
p= & \frac{1}{4}\left[\frac{3 \mu \rho_{0}^{2}}{8}-2 \mu+4 \beta \sin (\tau / 2)-2 \gamma \cos (\tau / 2)\right], \\
q= & \rho_{0}^{4}+A_{1} \rho_{0}^{2}+A_{0}, \\
A_{1}= & \frac{16}{3\left(\frac{\mu^{2}}{64}-3 \lambda^{2}\right)}\left[\lambda \delta_{1}+\frac{\mu^{2}}{32}+\frac{1}{4}\left(3 \lambda+\frac{\mu}{4}\right) K_{1}-\frac{\lambda}{8} K_{2}\right], \\
A_{0}= & \frac{16}{9\left(\frac{\mu^{2}}{64}-3 \lambda^{2}\right)}\left[\frac{1}{4}\left(\alpha^{2}-\mu^{2}\right)-\delta_{1}^{2}+\frac{K_{1}}{2}\left(\mu-\delta_{1}\right)-\frac{K_{2} \delta_{1}}{2}\right. \\
& \left.\quad-\frac{1}{4}\left(\gamma^{2}+4 \beta^{2}-4 \gamma \beta \sin \tau\right)\right], \\
K_{1}= & \gamma \cos (\tau / 2)-2 \beta \sin (\tau / 2), \quad K_{2}=\gamma \sin (\tau / 2)-2 \beta \cos (\tau / 2) .
\end{aligned}
$$

From the Routh-Hurwitz criterion [19], the steady-state response is asymptotically stable if and only if $p>0$ and $q>0$ which keep the real parts of the eigenvalues negative. The stability domain of the non-trivial solutions $\rho_{0}$ given by Eq. (11), when parametric excitation and time-delayed position (resp. time-delayed velocity) are combined, in the plane $\left(\delta_{1}, \alpha\right)$ is shown in Fig. 7. We used the values of the parameters as $\mu=0.1, \rho_{0}=0.1$, and $\tau=\pi$, 
since for these values, the stability is clearly analyzed. Fig. 7(a) (resp. Fig. 7(b)) correspond to the case $\beta<\gamma$ (resp. $\beta>\gamma$ ). Two main conclusions are obtained: the plane is divided in three regions where two of them are stable and one is unstable, also, the stable domain increases when the time-delay control gain position is greater than the time-delay control gain velocity (see Figs. 7(a) and (b)).

\section{Conclusion}

In this paper, we studied the parametric resonance in Eq. (1) that involves the interaction of parametric excitation with time-delayed position and velocity. Our analytical results are based on slow flow system Eqs. (9) that were obtained using first-order averaging. We studied the condition of reducing amplitude vibration as well as the nonlinear response and the stability of the steady-state solutions and the bifurcations that accompanied response and stability changes: saddle-nodes, Hopf bifurcations. By the stability analysis of the parametric Rayleigh-Duffing system, we shown that considering either time-delayed velocity alone or time-delay position and velocity could be better to enhance the stability domain.

\section{Acknowledgements}

The first author M. Siewe Siewe indebted to the University of Pretoria for its financial support to do research work as a senior visiting Fellow and also indebted to the Department of Electrical, Electronic and Computer Engineering for hosting him to undertake part of this work.

\section{Figures captions}

Figure 1 : Boundary criterion for the effectiveness of the control of the oscillation amplitude; red colour (upper curve) $\beta=0.3$, blue colour (lower curve) 0.1 , the other parameters are $\mu=0.002 \lambda=-1, \delta_{1}=0.01, \gamma=0.25$.

Figure 2 : The effect of reducing the steady-state response corresponding to Eq. (11) with control parameter $\alpha$ obtained in Fig. 1. (a) Frequency-response and (b) force-response.

Figure 3 : Time-delayed position gain $\beta$ effect on response-surface curves given by Eq. (11): (a) $\beta=0.1$ and (b) $\beta=0.3$.

Figure 4 : (a) The effect of the parametric amplitude in the frequency-response of the system in the absence of the time-delay. (b) Force-response for two detuning parameter values. Here $\beta=0.1, \mu=0.002, \lambda=-1$, and $\gamma=0.25$.

Figure 5: (a) The effect of the position time-delay alone in the frequency-response of 
the system without parametric excitation and (b) the effect of the time-delayeded velocity alone in the frequency-response of the system without parametric excitation for $\tau=0.008$ and $\mu=0.8$.

Figure 6 : Effect of parametric perturbation added to the time-delayed term in the response of the system. (a) Frequency-response amplitude and (b) Force-response. The values of the parameters are $\tau=0.008, \mu=0.8, \beta=0.1, \lambda=-1$, and $\gamma=0.25$.

Figure 7: Stability of the non-trivial solution defined by Eq. (15) in the plane $\left(\delta_{1}, \alpha\right)$ for $\mu=0.1, \gamma=0.5$, and $\tau=\pi$. (a) $\beta=0.1$ and (b) $\beta=0.5$. 


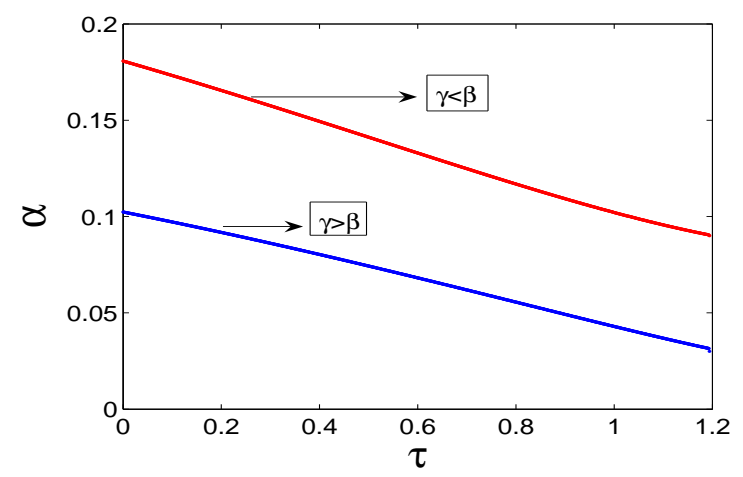

Figure 1:

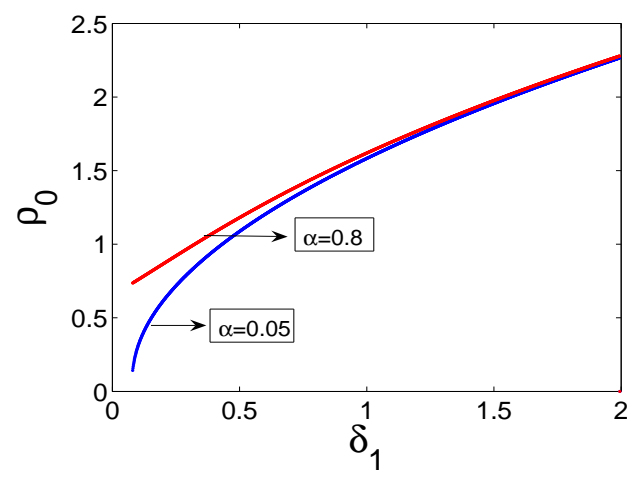

(a)

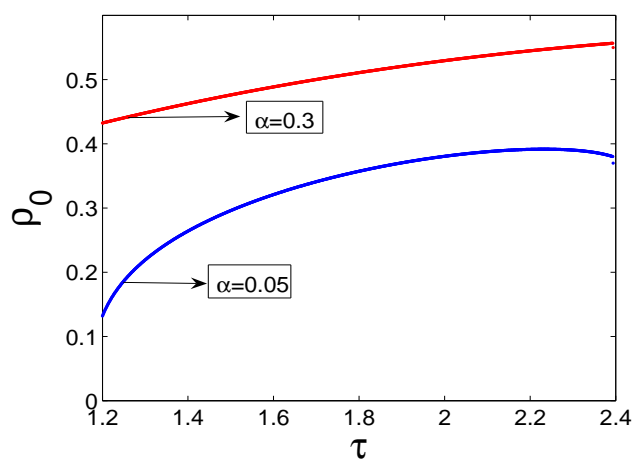

Figure 2: 


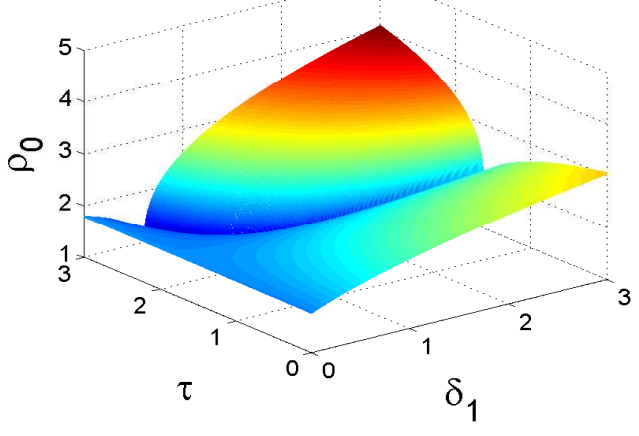

(a)

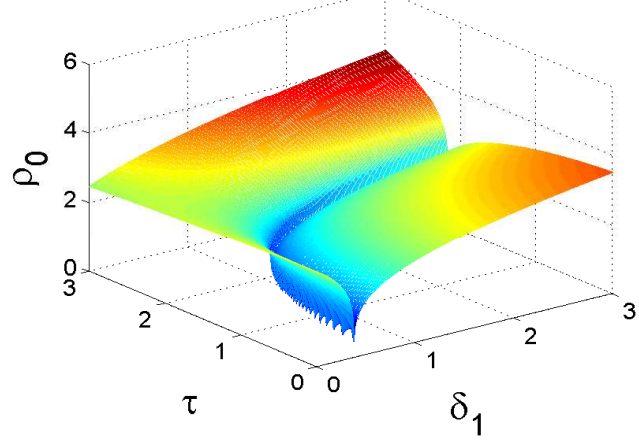

(b)

Figure 3:

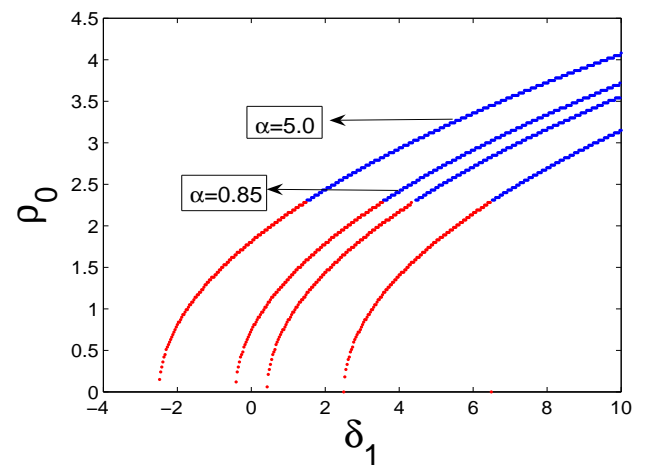

(a)

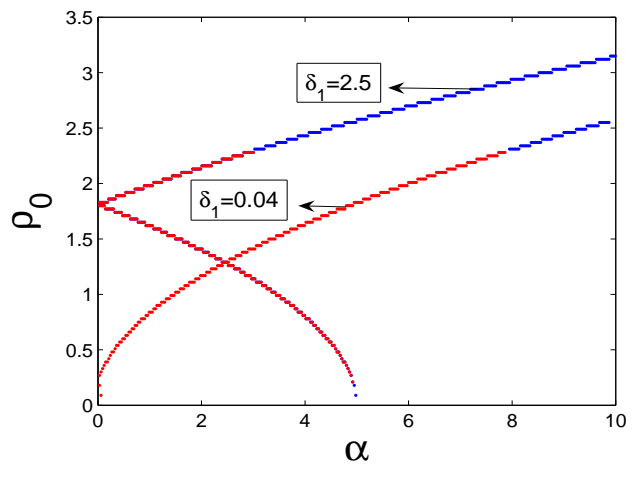

(b)

Figure 4:

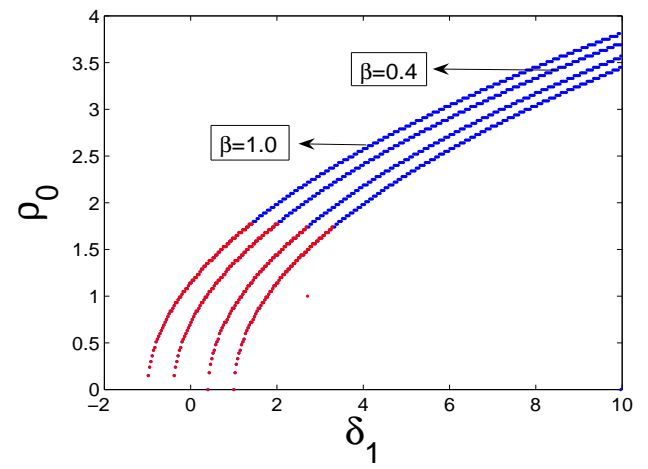

(a)

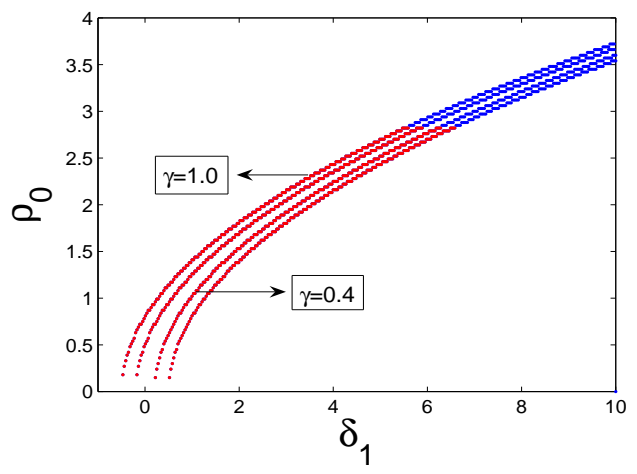

(b)

Figure 5: 

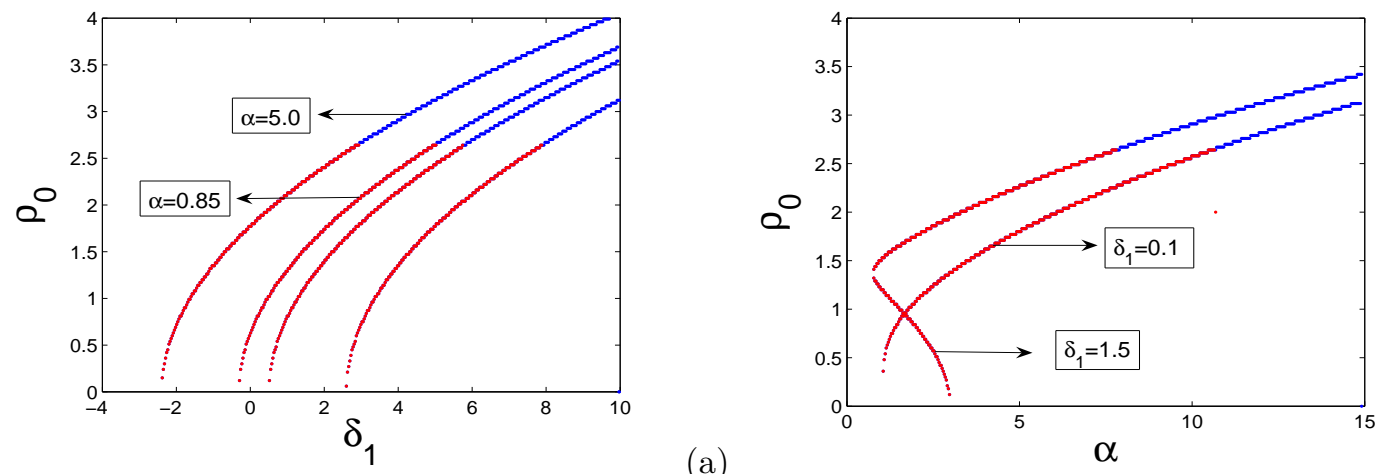

(a)

Figure 6:

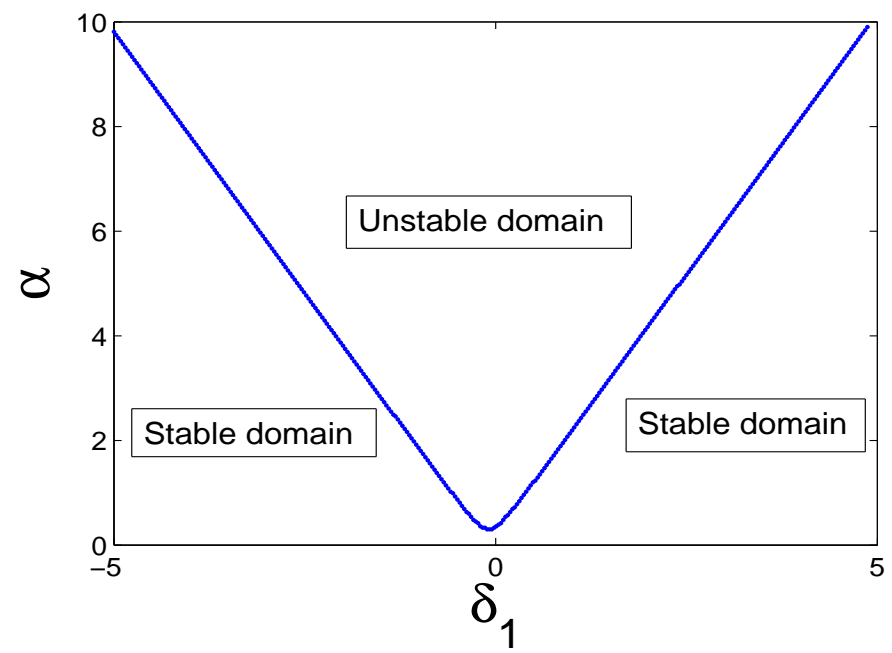

(a)

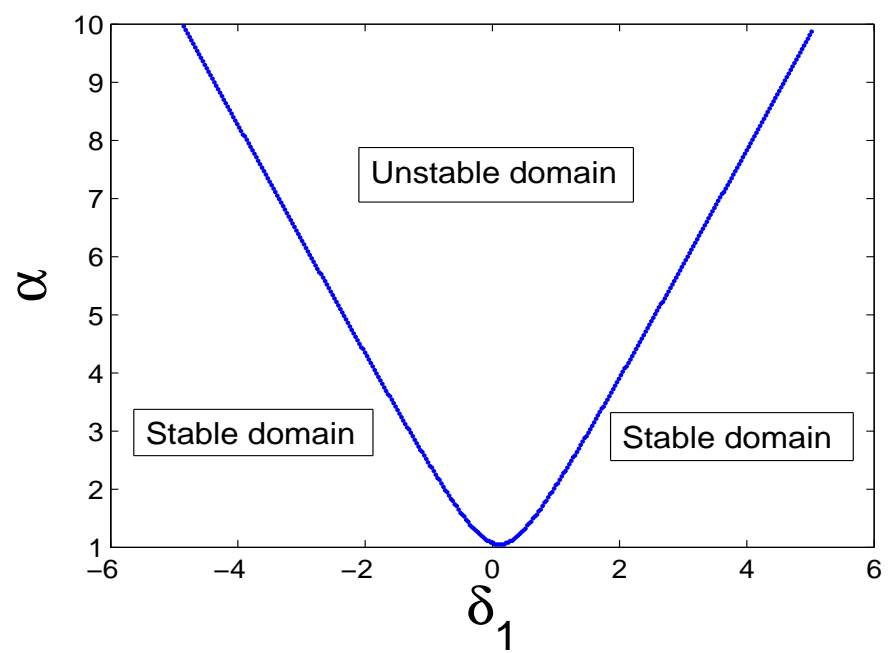

(b)

Figure 7: 


\section{References}

[1] S. A. Wirkus, The dynamics of two coupled van der Pol oscillators with delay coupling. Ph.D thesis, 1999.

[2] M. Lakshmanan and D.V. Senthilkumar, Dynamics of nonlinear time-delay systems. Springer, Berlin, 2010.

[3] Shayer L., and Campbell S. A., Stability, bifurcation, and multi-stability in a system of two coupled neurons with multiple time delays. SIAM J Appl Math 61:673-700, 2000.

[4] Liao X.F., and Chen G.R., Local stability, Hopf and resonant codimension-two bifurcation in a harmonic oscillator with two time-delays. Int J Bifur Chaos 11:2105-2121, 2001.

[5] Xu J., Yu P., Delay-induced bifurcations in a nonautonomous system with delayed velocity feedbacks. Int J Bifur Chaos 14:2777-2798, 2004.

[6] Ramana Reddy D. V., Sen A., and Johnston G. L., Time delay effects on coupled limit cycle oscillators at Hopf bifurcation. Physica D 129:15-34, 1999.

[7] Mackey M., and Glass L., Oscillation and chaos in physiological control system. Science 197:287-289, 1977.

[8] Aubin, K., Zalalutdinov, M., Alan, T., Reichenbach, R. B., Rand, R. H., Zehnder, A., Parpia, J. and Craighead, H. G., Limit Cycle Oscillations in CW Laser-Driven NEMS, Journal of Micro-electrical mechanical System 13:1018-1026, 2004.

[9] Zalalutdinov, M., Olkhovets, A., Zehnder, A., Ilic, B., Czaplewski, D. and Craighead, H. G., Optically pumped parametric amplification for micro-mechanical systems, Applied Physics Letters 78:3142-3144, 2001.

[10] Stepan, G., Insperger, T. and Szalai, R., Delay, Parametric excitation, and the nonlinear dynamics of cutting process, Int J Bifur Chaos 15:2783-2798, 2005.

[11] Kalmar-Nagy, T., Subcritical Hopf bifurcation in the delay equation model for machine tool vibrations, Nonlinear Dynamics 26:121-142, 2001.

[12] Rand, R. H., Ramani, D. V, Keith, W. L. and Cipolla, K. M., The quadratically damped Mathieu equation and its application to submarine dynamics, Control of Vibration and Noise: New Millennium 61:39-50, 2000.

[13] Wirkus, S., Rand, R. H. and Ruina, A., How to pump a swing, The College Mathematics Journal 29:266-275, 1998.

[14] Yu, M. F., Wagner, G. J., Ruoff, R. S. and Dryer, M. J., Realization of parametric resonances in a nanowire mechanical system with nanomanipulation inside a SEM, Physical Review Letters B 66: 235119-235124, 2002. 
[15] Zhang, C. Y., Zhu, Z. Q., Lin, Z. Q. and Wu, T. X., Theoretical and experimental study on the parametrically excited vibration of mass-loaded string, Nonlinear Dynamics 37:1$18,2004$.

[16] Xin-ye L., Yu-shu C., Zhi-qiang W. and Tao S., Response of parametrically excited Duffing-van der Pol oscillator with delayed feedback, Applied Mathematics and Mechanics 27:1585-1595, 2006.

[17] Attilio M., The Response of a Parametrically excited van der Pol Oscillator to a timedelay state feedback, Nonlinear Dynamics 26:105-119, 2001.

[18] Simohamed S, and Mohamed B., Effect of vertical high-frequency parametric excitation on self-excited motion in a delayed van der Pol oscillator, Chaos, Solitons and Fractals 37:1489-1496, 2008.

[19] Guckenheimer J., and Holmes P., Nonlinear oscillations, dynamical systems, and bifurcations of vector fields. New York: Springer- Verlag; 1983.

[20] Li S., Yang S., Guo W., Investigation on chaotic motion in histeretic non-linear suspension system with multi-frequency excitations, Mech. Res. Commun. 31:229-236, 2004.

[21] Grzegorz L., Marek B., Michael I. F., Kazimierz S., Chaotic vibration of a quarter-car model excited by the road surface profile, Communications in Nonlinear Science and Numerical Simulation 13:1373-1383, 2008.

[22] Zhehe Y.,Deqing M., Zichen C., Chatter suppression by parametric excitation: Model and experiments, Communications in Nonlinear Science and Numerical Simulation 330:2995-3005, 2011.

[23] Raghavendra D. N., Pravin M. S., Resonance, stability and chaotic vibration of a quarter-car vehicle model with time-delay feedback, Communications in Nonlinear Science and Numerical Simulation 16:3397-3410, 2011. 